\section{Clean Room Microscopy}

Walter C. McCrone ${ }^{1}$ and James A. Hertrich McCrone Associates, Inc.

Small particle contamination analysis requires consideration of secondary contamination of our sample. Protection of such samples is very important. The procedure for sampling with freshly pulled lengths of clear $3 \mathrm{M}$ Magic $^{\mathrm{TM}}$ tape is a favorite procedure among forensic microscopists. The tape must be immediately pressed onto a clean surface; usually, a clean microscope slide. There it is protected from further contamination.

Microscopical study and identification is usually possible utilizing the tape backing as a coverslip. Its thickness is about $56 \mu \mathrm{m}$, one-third of the recommended $170 \mu \mathrm{m}$, but quite adequate for objectives $4-20 \mathrm{X}$. If you feel persnickety, you can use two thicknesses of the tape, a second one over the sampling tape. This will give you optimum images for $4-40 \mathrm{X}$ objectives. Three thicknesses of tape, at $168 \mu \mathrm{m}$, is very close to the recommended thickness of $170 \mu \mathrm{m}$ for any or all objectives.

Air bubbles between the slide and tape sample may be a problem. They can be completely eliminated by placing a tiny droplet of benzene or xylene near one end of the slide. Then, holding a free end of the tape on the slide just before the droplet, "squeegee" the tape onto the droplet and slide with your finger pressed tightly on the tape as you squeegee to the far end of the tape. Very little of the added liquid remains between the tape and the glass and the refractive index around the particles is close to the tape index (about 1.53). Figure 1 shows an example of a particle collection taken from the top of a TV monitor in an office.

Individual particles are removed when necessary from such tapes by using a razor blade to cut a tiny square of tape immediately adjacent to the particle boundaries. This square (or rectangle) is removed with a sharpened steel or No.1 tungsten needle and then placed in a small droplet of benzene on a clean slide to dissolve the tape adhesive. The particle is pushed out of the droplet and along the clean slide surface to leave behind the benzene solution and tape square.

To remove all of the particles from the tape, from the slide it is placed in benzene in a centrifuge tube. After spinning down the suspension, the particles are pipetted out of the tube ignoring the undissolved tape backing.

Although these procedures are effective in avoiding sample contamination, it is also worth considering a simple clean bench. This avoids the high cost of a clean room, and it furnishes complete protection for samples, microscope, and all accessories within the bench. The clean bench (Figure 2) was designed and built by James Hertrich and used by McCrone Associates during the early days when we were using rented space and a clean room

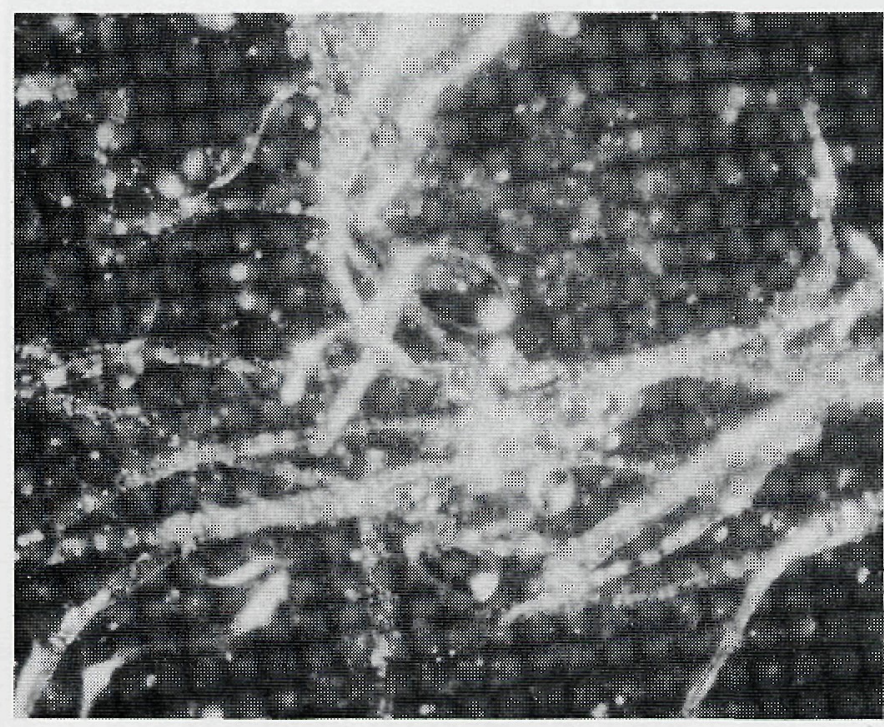

Figure 1: Photograph of a tape-sample from the top of an office PC monitor, 200X was not feasible. Each microscopist could have his or her own clean bench.

Microscopical examination of small particles requires care at all stages to prevent contamination of the sample. An exposed, clean microscope slide collects hundreds of easily visible dust particles/ $\mathrm{ft}^{2} / \mathrm{hr}$. A sample for microscopical study, exposed to the laboratory atmosphere is soon contaminated and it quickly becomes impossible to differentiate contamination from sample. Aside from minimizing contamination, clean facilities are also very pleasant to work in. One's microscope and work space remain substantially dustfree.

Lack of clean facilities requires fast work or covering the sample. In any case, a sample for microscopical examination should be opened and examined preliminarily under as much magnification as is practical, i.e., a loupe or, better, a stereobinocular microscope. The sample may be a failed relay; an integrated circuit, a speck in paper, film, tape, or some other substrate. An attempt should be made to locate the source of the trouble - the sample - and to characterize it as quickly as possible before any contamination can occur. Hopefully, subsequent contamination will be recognized as such.

Obviously, it is advantageous not to have to worry about sample contamination. A first step toward a clean room is simply a "clean box" (glove-box) or even a clean plastic bag. A microscope and the sample can be placed in the box (or bag). Clean nitrogen gas can be used to flush dust from the space before the sample is opened. If the sample is then encapsulated (in collodion, tape or some other suitable material), it can be removed for additional study.

Because the use of clean boxes and bags is awkward, a laminar-flow clean bench (Figure 1) is much better. In many ways, the clean bench is preferable to the clean room. Access to the room is easier, and less personnel time is involved in washing, changing clothes and cleaning. These advantages are achieved with no loss of dustfree air where it is essential - around the sample. The microscope, because it is in the clean bench, is also kept clean. Only the microscopist's hands and head extend into the clean space, and the 100 or more linear ft./min. laminar flow of clean air toward the front minimizes contamination from that source. An absolute (HEPA) filter forms the entire back wall of the bench. We have used the Cambridge Filter Corporation 1D-970-2 filter, but other units are commercially available. The bench is floor-mounted and connected to the wall through sponge rubber strips. Vibration from the motors, blowers, and air conditioner is thus minimized and dust is excluded.

The single unit is simple and easy to construct. The construction material is wood or transparent polymethyl methacrylate (PMMA) but all wood surfaces inside and out, must be covered with Formica®, Tygon® paint or the equivalent. The PMMA hood sidewalls and top help in lighting the interior and present a more

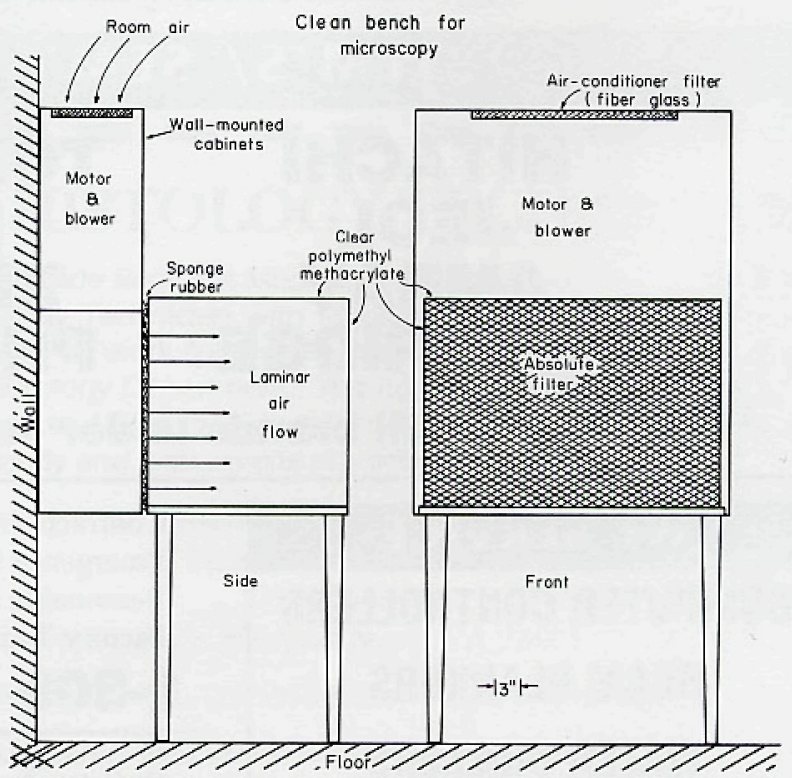

Figure 2: Single unit clean bench for microscopy; the motor is $1 / 3 \mathrm{hp}$ and the blower is a $330 \mathrm{cfm}$ "squirrel cage." 
pleasant working environment.

Tests have been made on all of the clean benches for air flow velocity and numbers of particles per $\mathrm{ft}^{3}$. An anemometer was used to measure the air flow velocity. The average for all benches was $130 \mathrm{cfm}$, and the range was from 110 to $160 \mathrm{cfm}$. The filters are usually furnished in a wooden box with a rubber "weather strip" all around the back edge. It is, of course, essential that this backing serve its designed purpose of completely sealing the filter from air leaks around the sides. The dust level in the clean bench was measured with a standard air sampler fitted with membrane filters. The latter must stop particles $0.8 \mu \mathrm{m}$ or less in diameter.

When properly set up, a clean bench of the type proposed here should easily fulfill the conditions for a Class 10,000 clean room, i.e., a maximum of 65 five-micron particles per $\mathrm{ft}^{3}$ under operation conditions. Our benches tested in the range of 10-30 five-micron particles per $\mathrm{ft}^{3}$ when new. After considerable use of the bench, the number of five-micron particles rises and often exceeds $65 / \mathrm{ft}^{3}{ }^{3}$ unless careful, complete, and regular clean-up procedures are instituted and unless the bench is kept clear of all unnecessary materials.

An effective cleanup procedure is to spray or paint the entire interior of the clean bench with an aqueous latex, e.g, Vultex 1-V-10, supplied by General Latex and Chemical Corporation. This material dries in minutes to a coherent, elastic film that can be easily peeled from any surface, carrying with it any loosely adhering dust particles.

If a high dust level persists - above, say, 10 five-micron particles per $\mathrm{ft}^{3}$ of air - the rubber strip seal between the wall and the PMMA hoods should be checked. If the seal is not perfect, the flow of clean air past the openings will aspirate dust-laden room air from around the back of the bench.

If constructed carefully, assembled properly and thoroughly cleaned at the start, the clean benches should operate as a Class 10,000 or better work space. Depending on the uses to which the bench is put, it should be cleaned with the liquid latex monthly or at somewhat longer intervals. Incidentally, nearly any apparatus, tools or even hands can be effectively cleaned with the latex suspension.

We have found these clean benches invaluable in preventing sample contamination. The clean air environment also saves time in cleaning and maintaining microscopes, improves the quality of visual and photomicrographic work, keeps the microscopist happier (and healthier?) and exerts a salutary influence on neatness of notebooks and quality of the microscopy carried out.

1. Present Address: McCrone Research Institute, 2820 S. Michigan Avenue, Chicago, IL, 60616-3292; www address: http://www.mcri.org

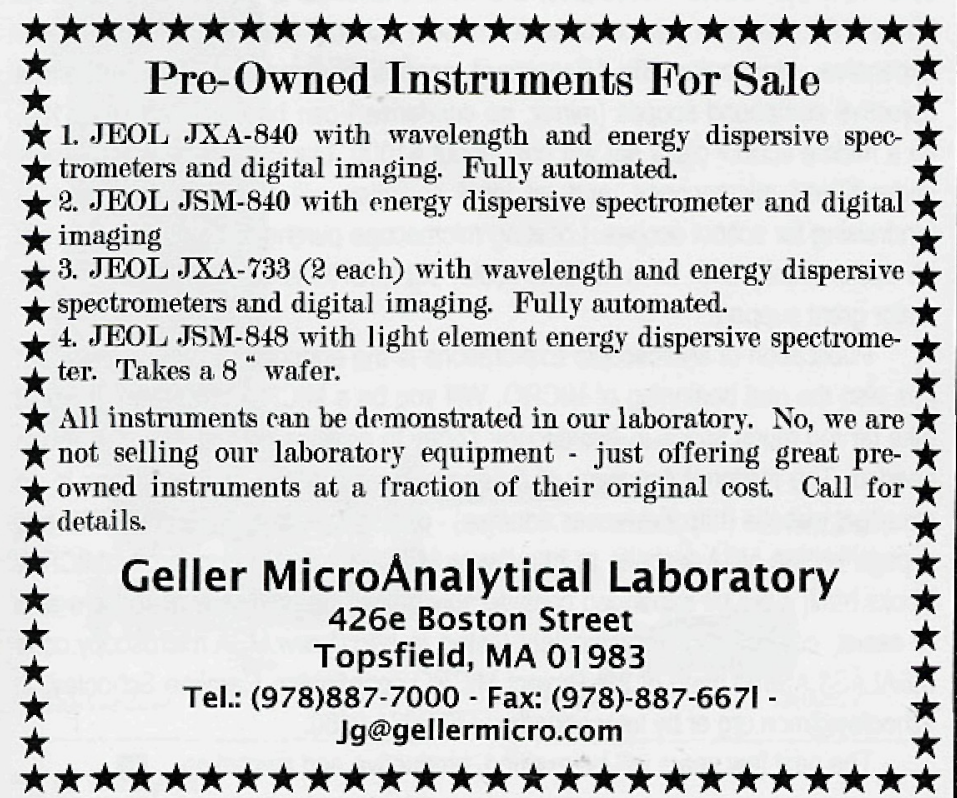

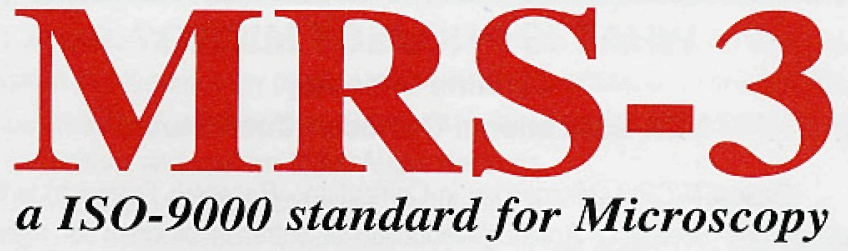

Our second generation, traceable, magnification reference standard for all types (SEM, Optical, STM, AFM, etc.) of microscopy. Usable from $10 \mathrm{X}$ to $50,000 \mathrm{X}$ with pitch patterns from $2 \mu \mathrm{m}$ $( \pm 0.1 \mu \mathrm{m})$ to $8 \mathrm{~mm}( \pm 0.25 \mu \mathrm{m})$. Pattern height traceable to $0.1 \mathrm{~mm}$ $\pm .001 \mu \mathrm{m}$. New patterns for parti-

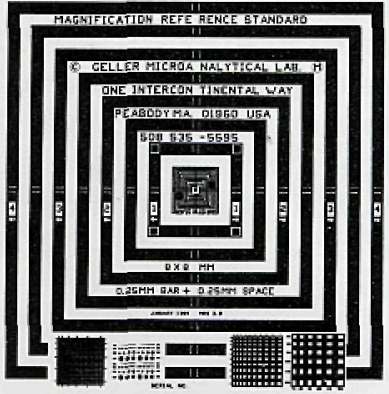
cle size counting (circles, squares and rectangles) and chemical imaging. Several hundred MRS's have been sold to date.

Send for our free RESOURCE GUIDE which discusses magnification measurement, error determination and calibration procedures.

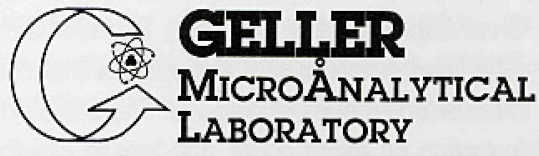

426e BOSTON STREET (RT. 1) * TOPSFTED, MA 01983 978/887-7000 * 978/887-6671* jg@gellermicro.com http://www.gellermicro.com

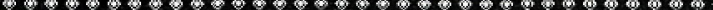

Certified to the ISO-9001 standard by Lloyd's Registry Quality Asssurance

"Doing it Right - the First Time"

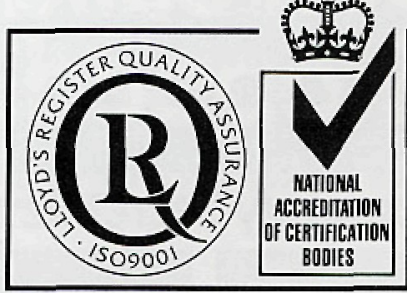

Analytical Services:

- Electron Probe SEM - Metallography Surface Analysis (ESCA \& Auger) Microhardness

Products:

- Elemental Reference Standards (some traceable)

- Magnification Standards (traceable)

- Vacuum Desiccators

- Computer Control System Updates for Electron Probes

- Digital Imaging for Analog SEMs

- Energy-dispersive electronics updates: pulse processor, pulse height analyzers and software (qualitative and quantitative)

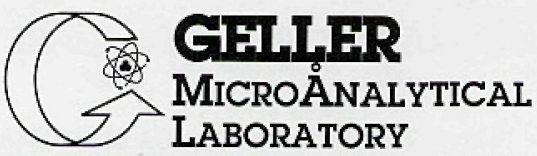

426e BOSTON STREET (RT. 1) * TOPSFTED, MA 01983

978/887-7000 *978/887-6671 * jg@gellermicro.com

http://www.gellermicro.com 